Original Article

\title{
Surgical Results of Completion Pneumonectomy
}

\author{
Shuji Haraguchi, MD, ${ }^{1,2}$ Kiyoshi Koizumi, MD, ${ }^{1,2}$ Tomomi Hirata, MD, ${ }^{1,2}$ \\ Kyoji Hirai, MD, ${ }^{1,2}$ Iwao Mikami, MD, ${ }^{1,2}$ Hirotoshi Kubokura, MD, ${ }^{1,2}$ \\ and Kazuo Shimizu, MD, FACS, FJCS ${ }^{2}$
}

\begin{abstract}
Purpose: We report our experience with completion pneumonectomy (CP).
Methods: We report on operative procedure and morbidity, mortality, and survival rates. Results: CP was performed for malignancy in 12 patients and postoperative complications after the first operation in 4 patients. Intrapericardial dissection of vessels was performed in 14 patients $(87.5 \%)$. Partial vertebrectomy from the second to the fifth vertebrae was performed in 1 patient. Carinal resection was performed in 2 patients. Morbidity including bronchopleural fistula, pulmonary insufficiency, pyothorax, and pulmonary infarction developed in 6 of the 16 patients (37.5\%). Mortality rate was 18.8\%. The actuarial 1-, 3-, and 5-year overall survival after CP for all malignancy was $80.8 \%, 49.0 \%$, and $49.0 \%$ respectively.

Conclusions: The morbidity and mortality rates are high in CP. Combined resection, especially carinal resection, appears to be contraindicated in CP. CP is one of the treatments of choice, even for malignancy, if complete resection is possible because of the good long-term survival.
\end{abstract}

Key words: lung cancer, completion pneumonectomy, mortality, morbidity.

\section{Introduction}

Completion pneumonectomy $(\mathrm{CP})$ refers to the resection of the remaining lung tissue after a previous pulmonary resection. Increased frequency of metachronous non-small cell lung cancer provides an indication to perform $\mathrm{CP}^{1}$; however, $\mathrm{CP}$ remains a risky procedure. ${ }^{2-14)}$ In the present study, we report our experience with $\mathrm{CP}$, considering the operative procedure, morbidity, mortality, and survival of patients.

\footnotetext{
${ }^{1}$ Department of Surgery, Division of Thoracic Surgery, Graduate School of Medicine, Nippon Medical School, Tokyo, Japan

${ }^{2}$ Department of Biological Regulation and Regenerative Surgery, Graduate School of Medicine, Nippon Medical School, Tokyo, Japan
}

Received: September 14, 2009; Accepted: February 3, 2010 Corresponding author: Shuji Haraguchi, MD. Department of Surgery, Nippon Medical School, 1-1-5 Sendagi, Bunkyo-ku, Tokyo 113-8603, Japan

Email: shuji@nms.ac.jp

(C)2011 The Editorial Committee of Annals of Thoracic and Cardiovascular Surgery. All rights reserved.

\section{Materials and Methods}

From April 1988 to September 2008, 17 patients including 13 males and 4 females underwent $\mathrm{CP}$ at the Division of Thoracic Surgery, Department of Surgery, Nippon Medical School and the Department of Surgery, Nippon Medical School Musashikosugi Hospital. Of these patients, 1 was excluded because she underwent resuscitation and rescue $\mathrm{CP}$ for massive hemoptysis due to local recurrence of lung cancer. Therefore, 16 patients with a mean age of 62 years (range, 37-80 years) were analyzed in the present study (Table 1). They accounted for $11.6 \%$ of all pneumonectomies performed during the same period. Nine and 7 patients underwent right and left CP, respectively. Fifteen patients (94\%) underwent lung resection for primary lung cancer and 1 patient $(6 \%)$ underwent lung resection for pulmonary tuberculosis at the first operation. According to the UICC staging system, ${ }^{15)} 8$ patients had stage I disease, 3 had stage II disease, and 5 had stage III disease. The procedures were performed through posterolateral thoracotomy in 9 patients, video-assisted thoracic surgery (VATS) in 2, 
Table 1 Clinical characteristics of 16 patients who underwent completion pneumonectomy.

\begin{tabular}{llllllll}
\hline Patient & Age \& Sex & Side & 1st Disease & 1st Approach & 1st Procedure & 2nd Disease & 2nd Approach \\
\hline 1 & $45 \mathrm{M}$ & Right & LC (IB) & PL & RUL + ND2a & Second LC (IIB) & PL \\
2 & $52 \mathrm{~F}$ & Left & LC (IB) & MS & LUL + ND2a & Second LC (IIIB) & Bilateral PL \\
3 & $55 \mathrm{M}$ & Left & LC (III) & PL & LUL + ND2a & Pulmonary infarction & PL \\
4 & $64 \mathrm{M}$ & Right & Tbc & PL & RUL & LC (III) & AL \\
5 & $50 \mathrm{M}$ & Right & LC (IB) & PL & RUL + ND2a & Local recurrence of LC & PL \\
6 & $58 \mathrm{M}$ & Right & LC (III) & PL & RUL + ND2a & Pulmonary metastasis & MS + AL \\
7 & $65 \mathrm{M}$ & Right & LC (IIIA) & MS & RUSL + ND3a & BPF & PL \\
8 & $78 \mathrm{M}$ & Right & LC (IB) & PL & RLL + ND2a & Pulmonary metastasis & PL \\
9 & $76 \mathrm{M}$ & Left & LC (IIB) & MS + AL & RUD + ND2a & Pulmonary metastasis & AL \\
10 & $52 \mathrm{M}$ & Left & LC (III) & AL & LLSL + ND2a & Broncho-PA fistula & AL \\
11 & $77 \mathrm{~F}$ & Left & LC (IA) & PL & LLL + ND2a & Second LC (IB) & MS + AL \\
12 & $63 \mathrm{M}$ & Right & LC (IIB) & PL & RMLL + ND2a & Pyothorax & MS + AL \\
13 & $65 \mathrm{M}$ & Right & LC (IIB) & PL & RLL + ND2a & Second LC (IA) & PL \\
14 & $37 \mathrm{M}$ & Right & LC (IA) & VATS & S8 seg + ND2a & Local recurrence of LC & PL \\
15 & $80 \mathrm{M}$ & Left & LC (IB) & MST & LLL + ND2a & Second LC (IA) & MS + AL \\
16 & $75 \mathrm{~F}$ & Left & LC (IB) & VATS & RUD + ND2a & Pulmonary metastasis & MST
\end{tabular}

M, male; F, female; LC, lung cancer; ( ), pathological stage of LC; Tbc, pulmonary tuberculosis; PL, posterolateral thoracotomy; MS, median sternotomy; AL, anterolateral thoracotomy; VATS, video-assisted thoracic surgery; MST, muscle-sparing thoracotomy; RUL, =right upper lobectomy; LUL, left upper lobectomy; ND, nodal dissection; RUSL, right upper sleeve lobectomy; RLL, right lower lobectomy; RUD, resection of upper division; LLSL, left lower sleeve lobectomy; LLL, left lower lobectomy; RMLL, right middle and lower lobectomy; seg, segmentectomy; BPF, bronchopleural fistula

median sternotomy in 2, and median sternotomy plus anterolateral thoracotomy, anterolateral thoracotomy, and muscle-sparing thoracotomy each in 1 patient. For the first operation, 13 patients (81\%) underwent lobectomy: right upper lobectomy in 4 , left lower lobectomy, left upper lobectomy, right lower lobectomy each in 2 , and right upper sleeve lobectomy, left lower sleeve lobectomy and right middle and lower lobectomy each in 1, and 3 underwent segmentectomy: resection of left upper division in 2 and right $S^{8}$ in 1 . Combined resection of the chest wall resection was performed in 1. Six patients had adenocarcinoma, 5 had squamous cell carcinoma, 2 had large cell neuroendocrine carcinoma, 1 had adenosquamous cell carcinoma, and 1 had carcinoid tumor.

Mortality was defined as death occurring during the hospitalization period following surgery. Morbidity in this study was defined as complications that occurred during the hospitalization period during and after surgery, and included respiratory failure necessitating mechanical ventilation for more than 48 hours, bronchopulmonary artery fistula, bronchopleural fistula (BPF), pyothorax, and bleeding.

\section{Results}

$\mathrm{CP}$ was performed for malignancy including second primary lung cancer in 5, metastatic lung tumor in 4, local recurrence in 2 , and primary lung cancer in 1 , and postoperative complications after the first operation in 4 patients (Table 1). The same approach with the prior thoracotomy was used in 7 patients (posterolateral thoracotomy: 5; and anterolateral thoracotomy: 2). Different approaches were used in 9 patients (median sternotomy plus anterolateral thoracotomy: 4; posterolateral thoracotomy: 2; bilateral posterolateral thoracotomy, muscle-sparing thoracotomy, and anterolateral thoracotomy each in 1). Intrapericardial dissection of vessels was performed in 14 patients (87.5\%). Partial vertebrectomy from the second to the fifth vertebrae was performed in patient 5. Carinal resection was performed in 2 patients (patients 2 and 6). Carinal resection, interrupted anastomoses, and closure of the left bronchial stump were performed by right posterolateral thoracotomy, and left completion pneumonectomy by left posterolateral thoracotomy in patient 2 because she had undergone left upper lobectomy by median sternotomy before. Carinal resection, interrupted anastomoses, and right completion pneumonectomy were performed by median sternotomy and anterolateral thoracotomy in patient 6 . Mean operating time was $398 \pm 166 \mathrm{~min}$. Mean volume of blood loss was $2423 \pm 2888 \mathrm{ml}$. Mean duration of hospital stay after surgery was $74 \pm 93$ days (Table 2). 
Table 2 Operative procedures, short and long-term surgical results of 16 patients.

\begin{tabular}{llllllll}
\hline Patient & $\begin{array}{l}\text { Combined } \\
\text { Resection }\end{array}$ & $\begin{array}{l}\text { Intrapericardial } \\
\text { dissection }\end{array}$ & $\begin{array}{l}\text { Operating } \\
\text { time }(\mathrm{min})\end{array}$ & $\begin{array}{l}\text { Blood loss } \\
(\mathrm{ml})\end{array}$ & Morbidity & $\begin{array}{l}\text { Postoperative } \\
\text { hospital stay (day) }\end{array}$ & $\begin{array}{l}\text { Survival } \\
\text { (month) }\end{array}$ \\
\hline 1 & None & Done & 510 & 2107 & None & 38 & 29D \\
2 & Carinal resection & Done & 840 & 1730 & BPF & 106 & 3D \\
3 & None & Done & 300 & 335 & None & 95 & 17D \\
4 & None & Done & 370 & 1990 & None & 23 & 20D \\
5 & Vertebrectomy & Done & 585 & 2000 & None & 58 & 11D \\
6 & Carinal & Done & 250 & 2000 & Pulmonary & 1 & 0D \\
7 & resection & & & & infarction & & 36A \\
8 & None & Done & 420 & 1755 & BPF & 256 & 21D \\
9 & None & Done & 347 & 1640 & RI & 63 & 36A \\
10 & None & Done & 500 & 2560 & None & 58 & 29A \\
11 & None & None & 209 & 1250 & None & 43 & 23A \\
12 & None & Done & 250 & 490 & None & 21 & 169A \\
13 & None & Done & 380 & 4750 & Pyothorax & 289 & 40A \\
14 & None & Done & 214 & 110 & None & 40 & 0D \\
15 & None & Done & 448 & 1400 & None & 23 & 3A \\
16 & None & Done & 495 & 12500 & RI & 1 & 19 \\
\hline
\end{tabular}

$\mathrm{BPF}$, bronchopleural fistula; RI, respiratory insufficiency; PA, pulmonary artery; D, dead; A, alive

Morbidity developed in 6 of the 16 patients (37.5\%); BPF and pulmonary insufficiency each in 2 patients, and pyothorax, pulmonary infarction each in 1 patient. BPF developed in 2 patients who underwent $\mathrm{CP}$ with carinal resection (patient 2) and $\mathrm{CP}$ for $\mathrm{BPF}$ after right upper sleeve lobectomy (patient 7), respectively. Bronchial anastomoses were covered with pedunculated precordial fat (patient 2) and intercostal muscle (patient 7) at the first operation. BPF was covered with omental flap and fenestration of left pleural cavity was added in patient 2 , but the patient died of massive bronchial bleeding 106 days after CP. Bronchial stump after CP covered with pedunculated intercostal muscle in patient 7 developed sutural insufficiency. He recovered after fenestration and thoracoplasty, but finally died of lung cancer.

Three patients (18.8\%) died during hospitalization because of pulmonary thromboembolism 1 day after $\mathrm{CP}$ (Patient 6), massive bronchial bleeding after bronchopleural fistula 106 days after CP (Patient 2), and adult respiratory distress syndrome 1 day after $\mathrm{CP}$ (Patient 16). Two of the 3 patients underwent carinal resection (patients 2 and 6). Patient 6 suddenly developed severe hypoxia and hypotension during the procedure so that completion pneumonectomy was performed under a percutaneous cardiopulmonary support (PCPS). An autopsy revealed pulmonary thromboembolism, which occluded left main pulmonary artery. Perforation of the aorta occurred during dissection of the carina in patient 16 , which was closed under a PCPS.

During the follow-up period, 4 men died of lung cancer and 1 woman died of another disease. Eight patients $(6$ male and 2 female) were alive. The actuarial 1-, 3-, and 5-year overall survivals after $\mathrm{CP}$ for all malignancy was $80.8 \%, 49.0 \%$, and $49.0 \%$ respectively (Fig. 1).

\section{Discussion}

Recent advances in operative techniques, anesthesia, and postoperative care have remarkably decreased the morbidity and mortality after surgery for lung cancer in Japan. ${ }^{16)}$ However, the previous ${ }^{2-14)}$ and present studies revealed that CP still had a high morbidity of $18 \%-53 \%$ and mortality up to $20.9 \%$. The present study revealed that carinal resection seemed to be risky. Either a complication of the initial lung resection or adjuvant radiation therapy or persistence of infectious pulmonary disease was a risky indication for $\mathrm{CP}^{2)}$ Maneuvers such as intrapericardial blood vessel ligation, division of the bronchus first, local application of glues and hemostatic agents, and bronchial reinforcement were routinely used to avoid 


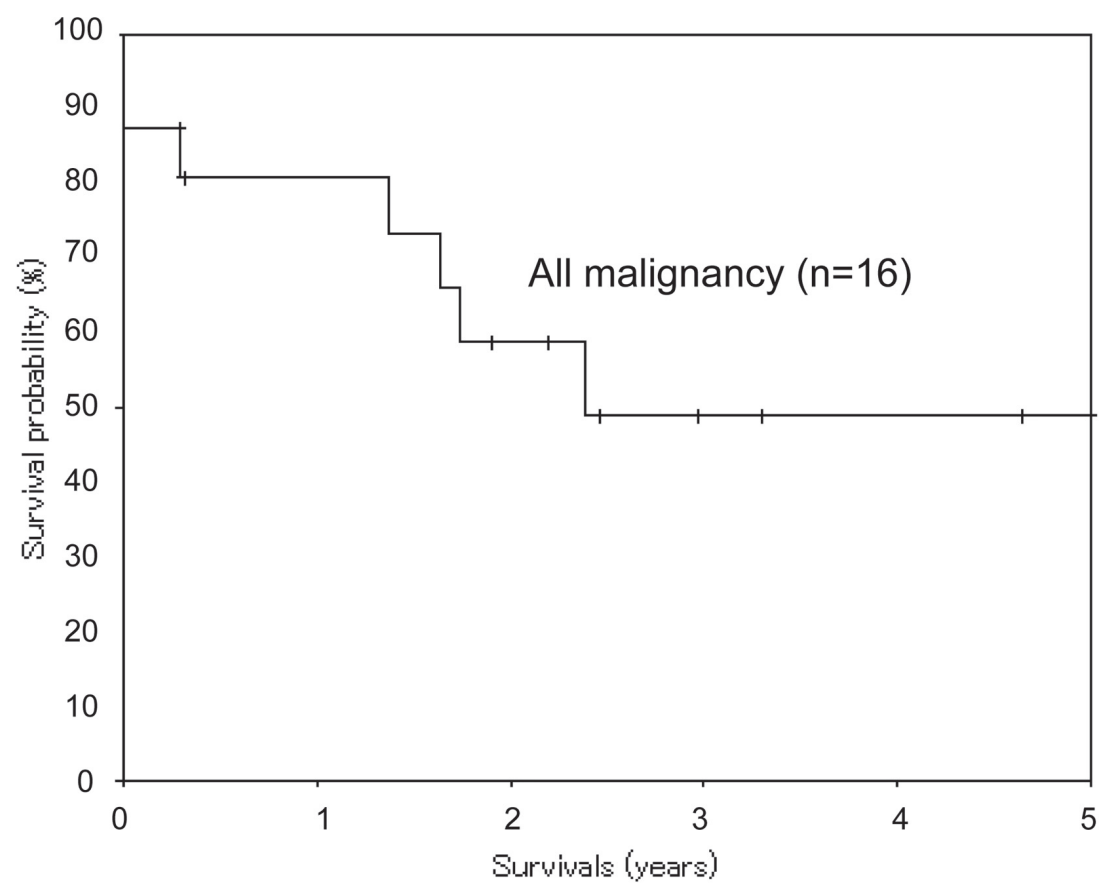

Fig. 1 Actuarial overall survival after completion pneumonectomy for all malignancy.

perioperative troubles. ${ }^{4,8)}$ Advanced age, preoperative corticosteroid use, or low operative hemoglobin, ${ }^{11)}$ and renal failure ${ }^{14)}$ were reported to be the significant risk factors based on the multivariate analysis. In the present study, no patient had preoperative corticosteroid use or renal failure. Two patients who underwent carinal resection died of bronchopleural fistula and pulmonary infarction, respectively. Patient 2 in the present study also underwent a left radical mastectomy and irradiation of 60Gy to the left parasternal area before left upper lobectomy for the first lung cancer. The second lung cancer developed in the area of the 2 nd to the 4th bronchial cartilage ring of the left main bronchus and seemed to be a radiation-induced carcinoma, and led to bronchopleural fistula. To our knowledge, resection of the carina has not been performed in previous studies. ${ }^{2-14)}$ Recently, various therapeutic bronchoscopy including laser therapy, electrocautery, brachytherapy, stents, and photodynamic therapy is reported to be useful in retrieving large airway obstruction. ${ }^{17)}$ Direct intratumoural injection with 5-fluorouracil is reported to reduce the size of tumors in the majority of cases and increase the diameter of the airway lumen. ${ }^{18)}$ Therefore, therapeutic bronchoscopy or the combination of bronchoscopic and surgical management should be performed to avoid carinal resection in these risky $\mathrm{CP}$ cases.
The actuarial 5-year overall survivals after $\mathrm{CP}$ for lung cancer have been reported to be $26.4 \%$ to $54 \% .^{2-14)}$ In the present study, the actuarial 5-year overall survivals after CP for all malignancy were $49.0 \%$. Accordingly, the good long-term survival of malignancy suggests that $\mathrm{CP}$ is one of the treatments of choice for selected patients if complete resection is possible.

In conclusion, the morbidity and mortality remain high in CP. Carinal resection appears to be contraindicated in CP. CP is one of the treatments of choice even for malignancy if complete resection is possible because of the good long-term survival.

\section{References}

1) Haraguchi S, Koizumi K, Hirata T, Hirai K, Mikami I, et al. Surgical treatment of metachronous non-small cell lung cancer. Ann Thorac Cardiovasc Surg 2010; 16: $319-25$.

2) McGovern EM, Trastek VF, Pairolero PC, Payne WS. Completion pneumonectomy: indications, complications, and results. Ann Thorac Surg 1988; 46: 141-6.

3) Oizumi H, Naruke T, Watanabe H, Sano T, Kondo H, et al. Completion pneumonectomy: a review of 29 cases. Nippon Kyoubu Geka Gakkai Zasshi 1990; 38: 72-7. (in Japanese)

4) Gregoire J, Deslauriers J, Guojin L, Rouleau J. Indica- 
tions, risks, and results of completion pneumonectomy. J Thorac Cardiovasc Surg 1993; 105: 918-24.

5) Al-Kattan K, Goldstraw P. Completion pneumonectomy: indications and outcome. J Thorac Cardiovasc Surg 1995; 110: 1125-9.

6) Massard G, Lyons G, Wihlm J-M, Fernoux P, Dumont $\mathrm{P}$, et al. Early and long-term results after completion pneumonectomy. Ann Thorac Surg 1995; 59: 196-200.

7) Terzi A, Furlan G, Terrini A, Magnanelli G. Completion pneumonectomy: experience with 47 cases. Thorac Cardiovasc Surgeon 1995; 43: 52-6.

8) Muysoms FE, de la Riviere AB, Defauw JJ, Dossche KM, Knaepen PJ, et al. Completion pneumonectomy: analysis of operative mortality and survival. Ann Thorac Surg 1998; 66: 1165-9.

9) Regnard JF, Icard P, Magdeleinat P, Jauffret B, Fares E, et al. Completion pneumonectomy: experience in eighty patients. J Thorac Cardiovasc Surg 1999; 117: 1095-101.

10) Fujimoto T, Zaboura G, Fechner S, Hillejan L, Schroder T, et al. Completion pneumonectomy: current indications, complications, and results. J Thorac Cardiovasc Surg 2001; 121: 484-90.

11) Miller DL, Deschamps C, Jenkins GD, Bernard A, Allen MS, et al. Completion pneumonectomy: factors affecting operative mortality and cardiopulmonary morbidity. Ann Thorac Surg 2002; 74: 876-84.

12) Guggino G, Doddoli C, Barlesi F, Acri P, Chetaille B, et al. Completion pneumonectomy in cancer patients: experience with 55 cases. Euro J Cardiothorac Surg 2004; 25: 449-55.

13) Sirmali M, Karasu S, Gezer S, Turut H, Findik G, et al. Completion pneumonectomy for bronchiestasis: morbidity, mortality and management. Thorac Cardiovasc Surg 2008; 56: 221-5.

14) Chataigner O, Fadel E, Yildizeli B, Achir A, Mussot S, et al. Factors affecting early and long-term outcomes after completion pneumonectomy. Euro J Cardiothoracic Surg 2008; 33: 837-43.

15) UICC. Lung and pleural tumours. In: Sobin LH, Wittekind Ch, eds.; UICC TNM Classification of malignant tumours. 5th ed., New York: John Wiley \& Sons, Inc., 1997; pp 91-100.

16) Kazui T, Wada H, Fujita H. Japanese Association for Thoracic Surgery Committee of Science. Thoracic and cardiovascular surgery in Japan during 2003 annual report by The Japanese Association for Thoracic Surgery. Jpn J Thorac Cardiovasc Surg 2005; 9: 517-6.

17) Lee P, Kupeli E, Mehta AC. Therapeutic bronchoscopy in lung cancer. Laser therapy, electrocautery, brachytherapy, stents, and photodynamic therapy. Lung Cancer 2002; 23: 241-56.

18) Celikoglu F, Celikoglu SI. Intratumoural chemotherapy with 5-fluoroouracil for palliation of bronchial cancer in patients with severe airway obstruction. J Pharm Pharmacol 2003; 55: 1441-8. 